

\title{
Using ultrasound to characterize the tongue-food interface: An in vitro study examining the impact of surface roughness and lubrication
}

Mathieu Mantelet, Frederic Restagno, Isabelle Souchon, Vincent Mathieu

\section{- To cite this version:}

Mathieu Mantelet, Frederic Restagno, Isabelle Souchon, Vincent Mathieu. Using ultrasound to characterize the tongue-food interface: An in vitro study examining the impact of surface roughness and lubrication. Ultrasonics, 2020, 103, pp.106095. 10.1016/j.ultras.2020.106095 . hal-02992276

\section{HAL Id: hal-02992276 \\ https://hal.science/hal-02992276}

Submitted on 6 Nov 2020

HAL is a multi-disciplinary open access archive for the deposit and dissemination of scientific research documents, whether they are published or not. The documents may come from teaching and research institutions in France or abroad, or from public or private research centers.
L'archive ouverte pluridisciplinaire HAL, est destinée au dépôt et à la diffusion de documents scientifiques de niveau recherche, publiés ou non, émanant des établissements d'enseignement et de recherche français ou étrangers, des laboratoires publics ou privés. 


\title{
Using ultrasound to characterize the tongue-food interface: An in vitro study examining the impact of surface roughness and lubrication
}

\author{
Mathieu Mantelet ${ }^{\mathrm{a}}$, Frédéric Restagno ${ }^{\mathrm{b}}$, Isabelle Souchon ${ }^{\mathrm{a}}$, Vincent Mathieu ${ }^{\mathrm{a}, *}$ \\ ${ }^{a}$ Université Paris-Saclay, INRAE, AgroParisTech, UMR SayFood, F-78850, Thiverval-Grignon, France \\ ${ }^{\mathrm{b}}$ UMR 8502 LPS, CNRS, Université Paris Sud, Université Paris-Saclay, Rue André Rivière, 91400 Orsay, France
}

\section{A R T I C L E I N F O}

\section{Keywords:}

Ultrasound

Apparent reflection coefficient

Tongue

Lubrication

Roughness

Food gels

\begin{abstract}
A B S T R A C T
We measured the apparent reflection coefficient of a 1-MHz ultrasound compressional wave at the interface between rough and lubricated tongue mimicking surfaces and various food gels, composed of agar or gelatin. For the smoothest mimicking surface, when a lubricating layer was present, the apparent reflection coefficient was fairly similar for the different food gels (33.6\% on average). The apparent reflection coefficient was significantly larger in the following situations: (i) tongue asperities were high and dense; (ii) lubrication levels were low; and (iii) gels were less rigid (range for the different gels-45.9-84.3\%). The apparent reflection coefficient conveys the ability of food gels to mold themselves to surface asperities or to form a coupling film of liquid at the interface. This study demonstrates that ultrasound methods can and should be used to explore the physical phenomena that underlie the texture perceptions resulting from tongue-palate interactions.
\end{abstract}

\section{Introduction}

To ensure a healthier food supply, public authorities are encouraging the food industry to reduce the sugar, salt, and fat content of processed foods. However, the success of the resulting food products will be determined by the sensory experience they create for the consumer. In particular, texture perception plays a major role in consumer preferences and choices. Therefore, to design healthier food products, we must have a better understanding of the mechanisms underlying texture perception.

\subsection{Oral processing and texture perception}

The International Organization for Standardization (ISO, 1992) defines food texture as "all the rheological and structure (geometric and surface) attributes of a food product, perceptible by means of mechanical, tactile, and where appropriate, visual and auditory receptors." During oral processing, food rheological and structural alterations are perceived by mechanoreceptors located in various organs of the oral cavity (including the lips, periodontal ligaments, oral mucosa, and tongue) [1-3]. Even though the use of these organs varies greatly among individuals and food categories [4], the tongue is the major organ responsible for evaluating food texture during oral processing [5]. The tongue is a highly complex organ-it is heterogeneous, viscoelastic, and anisotropic-and is composed of various groups of muscles that allow the food bolus to be handled with great maneuverability [6-9]. The contraction of these muscles during speech, food oral processing, or swallowing leads to (i) dramatic variation in the viscoelastic properties of the tissues and (ii) large-amplitude motion and changes in the shape and curvature of the dorsal surface of the tongue. Due to the presence of papillae (i.e., filliform, fungiform), the roughness of tongue surface may affect the mechanical interactions between the tongue and food during oral processing and, as a consequence, texture perception [10-12]. Various mechanoreceptors distributed throughout the lingual mucosa make it possible to evaluate and monitor the mechanical status of food during bolus formation [13]: slowly adapting (SA) receptors (Merkel's discs and Ruffini endings) handle static stimulations, and rapidly adapting (RA) receptors (Meissner's and Pacinian corpuscles) deal with the sudden onset/offset of stimuli. The activation of these receptors results in key tactile perceptions that not only influence the consumer's sensory experience but also help to define the most appropriate moment for triggering safe swallowing. More and more people are affected by swallowing disorders (dysphagia), which have potentially dramatic health consequences [14]; for example, there is an increased risk of choking and lung infections and, over the longer term, avoidance of certain foods and beverages, leading to malnutrition and/or dehydration. Even though disorders in motor ability or in oral movements may result in

${ }^{*}$ Corresponding author at: Paris-Saclay Food and Bioproduct Engineering Research Unit (SayFood UMR 782), 1 avenue Lucien Brétignières, 78850 ThivervalGrignon, France.

E-mail address: vincent.mathieu@inrae.fr (V. Mathieu). 
dysphagia issues independently from troubles in the sensorial system, the evaluation of texture perception during oral processing is essential to monitor the formation of the bolus and of its flow properties. The tribological characteristics of tongue-food interface can significantly influence bolus movements in the oral cavity and such frictional behaviors may drastically affect the solicitation of the mechanoreceptors inside tongue tissue, and then turn into specific texture perceptions. Consequently, it is essential to understand the mechanisms underlying texture perception that result from tongue-palate interactions in order to design healthy and desirable food products for people affected by dysphagia.

\subsection{Technical approaches for exploring texture perception}

There are various approaches for exploring the physical phenomena that take place between the tongue and palate and that define consumer perception of food texture [12]. Notably, rheology and tribology are two sub disciplines of mechanics that have served an essential role.

Prior to their introduction into the mouth, foods display complex rheological properties that can vary strongly over time, depending on the food's intrinsic stability, temperature, or manipulation by the consumer. The development of original model foods with controlled structural and rheological behaviors has thus become crucial for the understanding of texture perception between the tongue and the palate during oral processing $[15,16]$. For real products like cheese, the stress and strain at yield or fracture were found to be correlated with texture attributes like firmness, hardness, and elasticity [17]. In contrast, texture attributes such as creaminess, crumbliness, and graininess did not display this correlation because they are influenced by the mechanical breakdown of food during oral processing [12]. As a result of these relationships, various rheological methods have been proposed for studying the link between texture perception and the rheological properties of food boli (measured ex situ after expectoration by human experimental subjects or in vitro using devices developed to mimic oral processing) [18]. For example, such methods were successfully used to examine the relationships between the shear storage/shear loss moduli of food boli and the texture perception associated with mouth coating or residual sensations after swallowing [19]. However, there are limits to rheological approaches when it comes to studying texture attributes that depend on surface properties.

Fortunately, tribological approaches can be used to explore the friction mechanisms between food items and oral surfaces at the end of oral processing-these mechanisms influence texture attributes such as astringency, fattiness, and oiliness [12,18,20]. Tribology can also help researchers account for physiological properties of oral mucosa, such as roughness and lubrication. Such properties are as important as food properties when studying texture perception. However, at the same time, tribological approaches share an important constraint with rheological methodologies: they can only be applied to in vitro or ex vivo systems.

As mentioned before, texture perception is of multiparameter and dynamic nature, with multiple tissues and receptors involved [3,21]. Consequently, different review papers have suggested that it is important to develop in situ, real-time and non-invasive techniques for broadening our understanding of the impact of the pronounced variability in oral physiology on the mechanisms underlying texture perception $[12,18]$.

\subsection{Exploiting ultrasound techniques}

Because they are non-destructive and non-invasive, ultrasound (US) techniques show promise as a tool for characterizing the bulk and interfacial mechanical properties of food products and transformation processes [22-24]. US methods have thus been used in many fields of applications, such as the reconstitution of dairy powders [25], the detection of heterogeneities in various products (including cheese, chocolate, dough-based products as well as canned food) [26], the characterization of particle size in food emulsions [27], the monitoring of yogurt fermentation [28] as well as the investigation of wheat-flour water doughs [29]. The oral processing of food can also be viewed as a transformation process. US techniques could be developed to monitor the oral processing of food, which could yield novel insights into texture perception. An early study on US wave propagation across the tongue and oral cavity underscored the complexity of the resulting phenomena [30]. More recently, however, US imaging has nonetheless been used in myriad related contexts: to study the conformation of the tongue during speech [31], to understand the origin of sleep apnea events $[32,33]$, to study the swallowing phase in people suffering from dysphasia [34,35], and to investigate bolus flow during swallowing [36-41]. These studies demonstrate that US techniques can be applied to the tongue-food-palate system. Moreover, several studies have shown the utility of US imaging for monitoring the echo amplitude associated with the tongue dorsal surface, which allows tongue movement to be tracked during the oral processing of food $[37,42,43]$.

\subsection{Study aim}

The objective of this study was to investigate whether, theoretically, the amplitude of the echo from the dorsal surface of the tongue could reveal information about the physical interactions between the tongue and food. Such interactions are critical for the understanding of the mechanisms underlying texture perception. Given that wave propagation phenomena in the oral cavity are extremely complex, a controlled and highly simplified experimental framework was employed. More specifically, this study explored (i) how some specific properties of the tongue and of its environment (like surface roughness or the presence of a lubricating layer, considered independently from tongue movements and deformations) and (ii) how the properties of food could affect the apparent reflection coefficient of US waves at the interface between a tongue mimicking surface (hereafter referred as TMS) and food gels. Our results should help identify and characterize physical phenomena that may contribute to the stimulation of tongue mechanoreceptors during oral processing.

\section{Materials and methods}

\subsection{Model foods}

In this study, it was essential to use simple, homogenous model foods with controlled planar surfaces to create reproducible conditions. In past research, gels and emulsion-filled gels composed of agar and gelatin have proven useful when studying texture perception [44]. By varying the biopolymer concentrations in these products, it is possible to obtain substances with a broad range of mechanical properties (e.g., rigidity, adhesion, holding capacity), which result in different sensory experiences of texture. Consequently, such gels are helpful as models for investigating the utility of US techniques. Moreover, our research group recently characterized the US properties of these gels in a dedicated measuring cell made of polyvinyl chloride (PVC) with smooth surfaces [45]. We gained knowledge on the physical determinants of the apparent reflection coefficient between a smooth surface of PVC and model gels composed of agar and/or gelatin during sol-gel transition. These recent results will be helpful for now examining how the apparent reflection coefficient at the interface between similar model foods and a PVC tongue-mimicking surface varied for different degrees of roughness and lubrication.

Eight food gels composed of agar (HP700IFG, Kalys, Bernin, France) and/or gelatin (Bloom 250 PS 8/3, Rousselot, Gent, Belgium) were utilized. The preparation protocol was adapted from a previous study [44], and is described in detail elsewhere [45]. Briefly, the gelling agents and sucrose (Daddy, Cristalco, Paris, France) were dissolved in a solution of water (at $100{ }^{\circ} \mathrm{C}$ for agar, $60{ }^{\circ} \mathrm{C}$ for gelatin). The samples 
Table 1

Food gel composition (all contained 15\% wt $\%$ of sucrose).

\begin{tabular}{lllll}
\hline Food gel & Water (wt\%) & Agar (wt\%) & Gelatin (wt\%) & TWEEN 20 (wt\%) \\
\hline$A g_{0.3}$ & 84.7 & 0.3 & - & - \\
$A g_{1.8}$ & 83.3 & 1.8 & - & - \\
$G e_{3.5}$ & 81.5 & - & 3.5 & - \\
$G e_{7}$ & 78 & - & 7 & - \\
$\mathrm{Ag}_{0.3} G e_{3.5}$ & 81.2 & 0.3 & 3.5 & - \\
$\mathrm{Ag}_{0.7} G e_{5.85}$ & 78.45 & 0.7 & 5.85 & - \\
$\mathrm{Ag}_{1} G e_{0.75}$ & 84.25 & 1 & 0.75 & - \\
$G e_{7} T$ & 77.25 & - & 7 & 1.5 \\
\hline
\end{tabular}

were subsequently poured into cylindrical molds made of polyethylene (30 mm in diameter, $10 \mathrm{~mm}$ in depth) and left at $20^{\circ} \mathrm{C}$ for $15-18 \mathrm{~h}$. The cylindrical samples were individually unmolded at the last moment before the experiments began in order to prevent from sagging and syneresis phenomena which may occur over time.

Table 1 summarizes the composition of the eight model foods: two agar gels $\left(A g_{0.3}\right.$ and $\left.A g_{1.8}\right)$, three gelatin gels $\left(G e_{3.5}, G e_{7}\right.$, and $\left.G e_{7} T\right)$, and 3 agar-gelatin gels $\left(\mathrm{Ag}_{0.3} \mathrm{Ge}_{3.5}, \mathrm{Ag}_{0.7} \mathrm{Ge}_{0.75}\right.$, and $\left.A g_{1} \mathrm{Ge}_{0.75}\right)$. The $\mathrm{Ge} e_{7}$ and $\mathrm{Ge}_{7} \mathrm{~T}$ gels were the same except that $\mathrm{Ge}_{7} \mathrm{~T}$ contained TWEEN 20 (CAS: 9005-64-5 FG, Sigma-Aldrich, St Louis, MO, USA), an emulsifier that affects wettability.

The Young's modulus was measured for each food gel. Uniaxial compression tests were performed using a texture analyzer (TA.XT plus, Stable Micro Systems, Surrey, United Kingdom) on samples lubricated with paraffin oil. Lubricating the samples with paraffin oil had for aim to limit barreling effects caused by friction on the basis and on the top of the samples during the tests. Such phenomena may lead to undesired mechanical resistances, and consequently to the disturbance of elasticity measurements [44]. The experiments were carried out at room temperature $\left(20^{\circ} \mathrm{C}\right)$. A 70 -mm-diameter flat circular probe was used, employing a constant speed of $1 \mathrm{~mm} . \mathrm{s}^{-1}$ and a strain rate of up to $80 \%$. The Young's modulus values were determined using Matlab (MathWorks, Natick, Massachusetts, USA), calculated as the values of the initial slope of the stress/strain curve. At least six replicates were performed for each food gel.

\subsection{Tongue mimicking surfaces}

PVC cylinders (50 $\mathrm{mm}$ in diameter, $25 \mathrm{~mm}$ in height) were manufactured (UFP, Le Versoud, France) for use as TMSs. A non-deformable material was chosen so that the experiment could focus on surface properties and exclude changes due to material deformation. The diameter of the artificial tongues was set larger than the diameter of the model foods so that to ensure that the model foods fully rest on the TMS. As these experiments were conducted with a non-deformable material (PVC), changing the thickness would not have altered the mechanical behavior of the food samples in contact with the asperities of the TMSs. The thickness of the PVC cylinders was set after a series of tests so that to accommodate with the acoustic properties of the PVC. Consequently, the thickness was chosen (i) high enough to prevent from nearfield US measurements, (ii) low enough because of high attenuation of US waves in PVC, and (iii) so that to have a US beam diameter well adapted to the diameter of the food samples.

\subsubsection{Surface roughness}

Three different levels of surface roughness (referred to as $R_{0} \beta_{0}, R_{1} \beta_{1}$, and $R_{1} \beta_{2}$ ) were used to investigate how this variable affected US patterns at the interface between the TMSs and the food gels. $R_{o} \beta_{o}$ was the smoothest TMS, and it did not undergo any modifications after being machined by the supplier. The two rougher TMSs, $R_{1} \beta_{1}$ and $R_{1} \beta_{2}$, were obtained using similar methods. First, a sheet of sandpaper was stuck on a heating plate (RCT basic, IKA, Staufen im Breisgau, Germany), heated to $150{ }^{\circ} \mathrm{C}$, and coated with a thin film of vegetable oil (Carlex spray,
Zeelandia, Zierikzee, The Netherlands). The PVC cylinders were then placed on the sandpaper and compressed by a mass for a given duration of time. The molding duration and the amplitude of the normal load applied to print the asperities of the sandpaper were chosen after series of preliminary tests. The rougher the reference of the sandpaper, the higher the amplitude of the normal load and the duration required in order to ensure the achievement of a homogeneous and well molded surface. For each type of roughness, three different samples have been manufactured in order to verify the reproducibility of surface manufacturing. $R_{1} \beta_{1}$ and $R_{1} \beta_{2}$ differed in the following ways:

- (i) the type of sand paper used: a grain size of P60 was used for $R_{1} \beta_{1}$ (finishing paper P60, Klingspor, Haiger, Germany), and a grain size of P40 was used for $R_{1} \beta_{2}$ (Z40, Norton, Saint Gobain, Courbevoie, France)

- (ii) the mass used to compress the cylinders: $5 \mathrm{~kg}$ for $R_{1} \beta_{1}$ and $15 \mathrm{~kg}$ for $R_{1} \beta_{2}$

- (iii) the molding duration: $2 \mathrm{~h}$ for $R_{1} \beta_{1}$ and $10 \mathrm{~h}$ for $R_{1} \beta_{2}$

A stylus profiler, a Dektak XT (Bruker, Billerica, MA, USA), was then used to measure the roughness of the three surface profiles $\left(R_{0} \beta_{0}, R_{1} \beta_{1}\right.$, and $R_{1} \beta_{2}$ ). A stylus with 50 -nm-radius and 0.5-mg-strength was used. Vision64 $\mathrm{Map}^{\mathrm{TM}}$ software (Bruker, Billerica, MA, USA) was utilized to impose $60-\mathrm{mm} 1 \mathrm{D}$ scans and analyze the results. Three scans were performed for each profile (applying a $45^{\circ}$ rotation between scans) to obtain a value that was representative of the overall surface. A slope correction was applied prior to processing the roughness parameters with MATLAB $^{\circledR}$ (MathWorks, Natick, Massachusetts, US). Two classical parameters were chosen: the arithmetical mean height, $R_{a}$, which was the mean height of the asperities, and the correlation length, $\beta$, which was related to the density of the asperities [46]—the higher the $\beta$, the lower the density. The analysis of $1 \mathrm{D}$ scans obtained by profilometry measurements made it possible to assess (i) the spatial homogeneity of roughness properties on the samples taken individually, as well as (ii) the reproducibility of surface manufacturing (data not shown). Once the reproducibility of surface manufacturing confirmed, only one TMS of each type was kept for the study.

\subsubsection{Surface lubrication}

We examined how lubrication levels affected US patterns at the interface between the TMSs and the food gels. There were four levels: dry, low, medium, and high. For the sake of simplicity, water was used as the lubricant agent.

Under dry conditions, no lubricant was present. For the low and medium lubrication levels, the TMSs were sprayed with water. The two treatments differed in the amount of water used (water mass was weighed with a precision balance: Precisa 303A, Dietikon, Switzerland). For the high lubrication level, $2 \mathrm{~g}$ of water were poured onto the TMSs with a pipette, which ensured that their surfaces were completely submerged.

Table 3 summarizes, for each lubrication level, the mass of the water that was deposited and the thickness of the resulting film (assuming homogeneous spreading).

\subsection{Ultrasound measurements}

A US device and associated signal processing techniques were designed to investigate variation in the apparent reflection coefficient of US waves at the interface between the TMSs and the food gels.

\subsubsection{Experimental set-up}

A single-element piezoelectric transducer (V103RM, Olympus, Shinjuku, Tokyo, Japan) with a central frequency of $900 \mathrm{kHz}$ was used as both an emitter and a receiver (pulse-echo mode). This frequency was chosen to minimize the attenuation of the US waves by the PVC, as well as to ensure that measurements could be obtained beyond the 
near-field distance. The transducer was plugged into a US pulser-receiver (Sonatronic, Evry, France). The system emitted a negative squared pulse (width of $500 \mathrm{~ns}$, amplitude of $80 \mathrm{~V}$ ) to the transducer and digitalized (12-bit quantification, $100 \mathrm{MHz}$ sampling rate, $38 \mathrm{~dB}$ gain) the $r f$ signals. The settings for ultrasound emission and amplification have been defined based on preliminary experiments performed with a smooth PVC cylinder $\left(R_{O} \beta_{0}\right)$ surrounded by air. The observation of the echo from the interface between $R_{o} \beta_{0}$ and air made it possible to adjust the different parameters (width, voltage, gain) so that (i) to optimize the shape of the waveform, (ii) to find the suitable compromise between signal-to-noise ratio and attenuation properties of PVC and (iii) to avoid signal saturation. The data recording system did not make it possible to modify the sampling conditions (digitizer resolution and samplerate). However, the technical specifications of the system ensured (i) that the signals were sampled in compliance with the generalized Nyquist-Shannon sampling criterion, and (ii) that the resolution was high enough for the detection of low variations in the amplitude of ultrasound signals. The pulser-receiver was controlled using a dedicated user interface programmed with LabVIEW $^{\circledR}$ (National Instruments, Austin, Texas, USA).

The experiments were conducted in a climate-controlled room $\left(19{ }^{\circ} \mathrm{C}\right)$. The US transducer was placed beneath the lower face of the PVC cylinders (see Fig. 1) for at least $2 \mathrm{~h}$ before the experiments were begun to ensure that acoustic coupling (use of silicon grease) and temperature conditions had stabilized. The TMSs were cleaned prior to each measurement. The surfaces of the TMSs were brushed with dish soap (Liquide Vaisselle Main Ecologique, Prop, Paris, France) and water for $10 \mathrm{~s}$ and then rinsed three times with $2 \mathrm{ml}$ of water. This protocol was necessary to ensure (i) that the surface of PVC was properly cleaned from any residues of food gels between two experiments and (ii) that identical initial surface properties of the TMSs were recovered before starting a new test. However, it is important to note that the presence of soap residues on the surface of PVC may affect the properties of the TMSs by strengthening hydrophobicity, which is already important for PVC. As a consequence, the protocol was strictly and carefully followed in order to start from identical initial conditions.

First, reference US $r f$ signals were recorded immediately after cleaning the TMS, before the food gel was deposited. Second, one of the four lubrication treatments was applied to the TMS. Third, a food gel was removed from its mold and carefully placed at the center of the TMS. Finally, the US $r f$ signals of the TMS-food system were recorded. Measurements were obtained at least in triplicate for each set of conditions (i.e., for a given food gel on a TMS of a given roughness with a given lubrication level).

\subsubsection{Signal processing}

The $r f$ signals were processed using MATLAB ${ }^{\circledR}$ (MathWorks, Natick,
Massachusetts, US) in order to determine the apparent reflection coefficients of the US waves at the interface between the food gels and the TMSs (referred to as $R^{*}$ ). For all the $r f$ signals, high-frequency noise was reduced using a low-pass filter (15-MHz cut-off frequency) applied to the fast Fourier transform (FFT). The $r f$ signals were composed of an echo referred to as $E_{0}$, which corresponds to the acoustic energy reflected at the boundary of the TMS (see Fig. 1.b).

For each $r f$ signal, an automatic procedure was developed to extract a 4- $\mu$ s window centered on the maximum of the Hilbert transform of $E_{0}$. The FFT of this window was then calculated, and the amplitude $\left(A_{0}\right)$ of $E_{O}$ was defined as the amplitude of the FFT at $900 \mathrm{kHz}$ (the central frequency of the transducer). Amplitudes were measured in the frequency domain using a method developed for interfaces with partial contact [47] that displays low sensitivity to signal noise.

The apparent reflection coefficient of the US waves at the interface between the food gel and the TMS was then calculated as follows:

$R^{*}=100 * \frac{A_{0}}{A_{0, r e f}}$

where $A_{o, \text { ref }}$ is the amplitude of $E_{O}$ obtained on reference signals recorded before lubricating the TMS and positioning the food sample. Before placing the food gel, the only medium in contact with the TMS was air. Under such conditions, due to the negligible acoustic impedance of air (as compared to PVC), $E_{O}$ corresponded to the full reflection of the acoustic energy received by the TMS. $A_{O, \text { ref }}$ is thus a reference measurement of the maximum observable amplitude and is used as the basis for calculating the apparent reflection coefficient.

No significant variation in $A_{0, r e f}$ was observed across the three levels of surface roughness. The wavelength of the US waves in PVC and food (greater than $1.5 \mathrm{~mm}$ ) was much longer than the mean height of the roughest profile (around $60 \mu \mathrm{m}$ ). As a consequence, scattering phenomena taking place at the interface had a negligible impact on $A_{o, r e f}$.

\subsection{Statistical analysis}

Analyses of variance (ANOVAs) accompanied by post-hoc analysis (Newman-Keuls) were performed using R (R Core Team 2018, R Foundation for Statistical Computing, Vienna, Austria) on the Young's modulus values and the $R^{*}$ values; the latter were log transformed to meet the assumption of normality. The alpha level was 0.05 .

\section{Results and discussion}

\subsection{Impact of surface roughness on $R^{*}$}

The TMSs differed in surface roughness (Table 2). $R_{a}$ values were significantly different between $R_{O} \beta_{O}$ and both $R_{1} \beta_{1}$ and $R_{1} \beta_{2}$. There
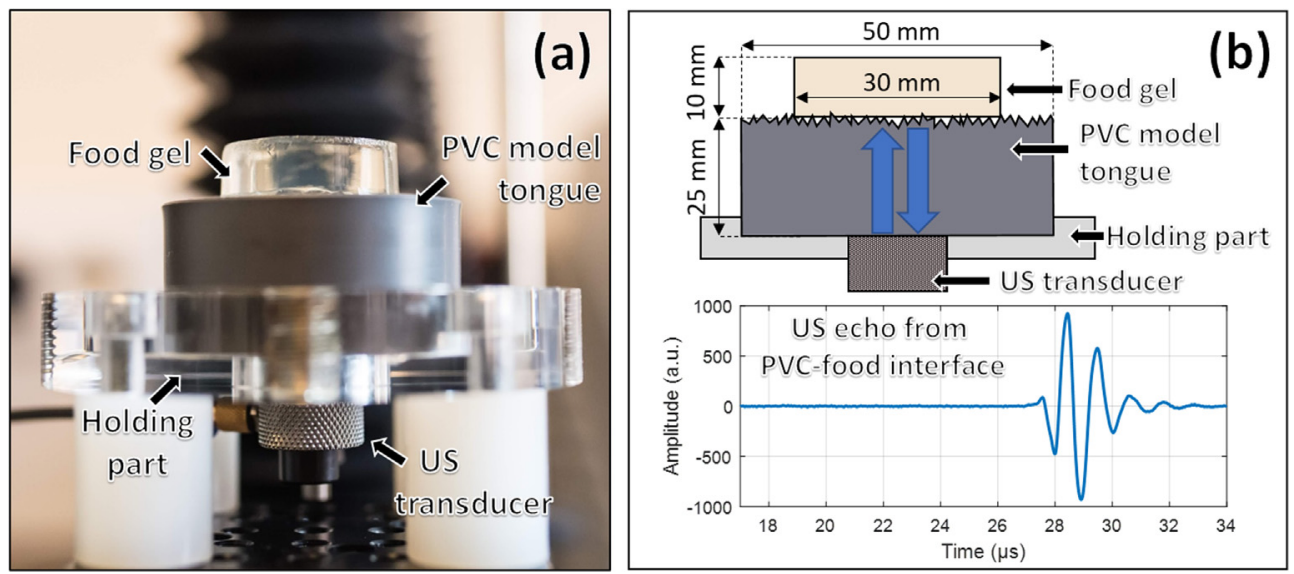

Fig. 1. (a) Experimental set-up and (b) Schematic representation of the set-up and a typical ultrasound (US) $r f$ signal. 
Table 2

Properties of the three tongue mimicking surfaces (mean \pm standard deviation) and the range of observed tongue asperity thickness in the literature. Letters following standard deviation values indicate mean values significantly differing among the four products (Newman-Keuls, $\mathrm{p}<0.05$ ).

\begin{tabular}{lll}
\hline Surface roughness level & $R_{a}(\mu \mathrm{m})$ & $\beta(\mu \mathrm{m})$ \\
\hline$R_{o} \beta_{O}$ & $2.54 \pm 0.09^{\mathrm{a}}$ & $35 \pm 0.4^{\mathrm{a}}$ \\
$R_{1} \beta_{1}$ & $58.2 \pm 5.3^{\mathrm{c}}$ & $206 \pm 4^{\mathrm{b}}$ \\
$R_{1} \beta_{2}$ & $52 \pm 11.2^{\mathrm{b}}$ & $243 \pm 12^{\mathrm{c}}$ \\
Range for human tongue & $40-100[48]$ & - \\
\hline
\end{tabular}

Table 3

Mass of water deposited on the tongue mimicking surfaces and the resulting thickness of the film formed (mean \pm standard deviation) for the four lubrication levels. The range of values for salivary film thickness observed in the literature is also indicated.

\begin{tabular}{lll}
\hline Lubrication level & Mass of water deposited $(\mathrm{mg})$ & Film thickness $(\mu \mathrm{m})$ \\
\hline Dry & 0 & 0 \\
Low & $30 \pm 5$ & $15 \pm 3$ \\
Medium & $86 \pm 9$ & $40 \pm 5$ \\
High & 2000 & 1000 \\
Range for human tongue & - & $12-54[70]$ \\
\hline
\end{tabular}

were also significant differences in $\beta$. $R_{0} \beta_{O}$ had the lowest values of $R_{a}$ and $\beta$, confirming that it was smoother than $R_{1} \beta_{1}$ and $R_{1} \beta_{2}$. The results also suggest that, in spite of the different sandpaper grain sizes used on $R_{1} \beta_{1}$ versus $R_{1} \beta_{2}$, the two TMS ended up with similar $R_{a}$ values. As a consequence, the apparent reflection coefficients for $R_{1} \beta_{1}$ and $R_{1} \beta_{2}$ were helpful in evaluating the impact of $\beta$. Prior research has revealed that the $R_{a}$ mean observed for real tongues ranges between 40 and $100 \mu \mathrm{m}$, which shows that $R_{1} \beta_{1}$ and $R_{1} \beta_{2}$ displayed physiological relevant values [48].

Fig. 2 shows the variations of the values $R *$ measured on the three types of TMSs $\left(R_{0} \beta_{0}, R_{1} \beta_{1}\right.$ and $\left.R_{1} \beta_{2}\right)$ and for three of the gels $\left(G e_{7}, G e_{3.5}\right.$, and $A g_{0.3}$ ). There was a significant effect of surface roughness on $R^{*}$ (overall ANOVA: p-value $\left\langle 10^{-3}\right.$ ). In this figure, the variations of $R^{*}$ as a function of surface roughness have been shown for different gels in order to illustrate that whatever the type of food product considered,



Fig. 2. Mean values and standard deviations of the apparent reflection coefficient at the interface between TMSs and food gels. The results were obtained under dry lubrication conditions, at the three roughness levels, and for three food gels: $\mathrm{Ge}_{7}, \mathrm{Ge}_{3.5}$ and $\mathrm{Ag}_{0.3}$. Letters indicate mean values significantly differing among the three products (Newman-Keuls, $\mathrm{p}<0.05$ ). similar tendencies were reported. For each type of food product taken separately, the highest values of $R^{*}$ were reported for $R_{1} \beta_{1}$, followed by $R_{1} \beta_{2}$, and lastly by $R_{0} \beta_{0}$. The impact of food properties for identical roughness properties was for its part discussed in Section 3.2. The values of $R *$ obtained with $R_{o} \beta_{0}$ can be compared to similar measurements performed between PVC and model gels in a previous study [45]. In that last case, the roughness of PVC surface was equivalent to $R_{o} \beta_{O}$ while the model foods were studied during sol-gel transition (from 50 to $\left.20{ }^{\circ} \mathrm{C}\right)$. The shear storage modulus and shear loss modulus values $\left(G^{\prime}\right.$ and $G^{\prime \prime}$, respectively) were characterized for very similar model foods and compared with different US properties. The apparent reflection coefficient was measured at the interface between a dedicated PVC cell with smooth surfaces (i.e., of identical smoothness to $R_{o} \beta_{O}$ ) and food gels. The food products were gelled directly in the cell, ensuring complete bonding between the food gels and the PVC (i.e., no air trapped at the interface). In this experimental set-up, the apparent reflection coefficient was solely determined by the acoustic impedance of the food gels and of the PVC. The slight differences in the apparent reflection coefficient observed across the food gels (range: $30-34 \%$ ) was primarily affected by variation in food compressibility (the bulk elastic modulus was greater than $2.5 \mathrm{GPa}$ ), and not by either of the shear moduli $\left(\mathrm{G}^{\prime}<50 \mathrm{kPa}, \mathrm{G}^{\prime \prime}<0.5 \mathrm{kPa}\right)$. Consequently, it appears that the viscoelastic properties of food gels have a negligible impact on gel acoustic impedance.

In this study, the apparent reflection coefficient obtained with $R_{o} \beta_{O}$ fell within the range observed in the previous study $(30-34 \%$; see Table 4 and Fig. 2), suggesting that the food gels were in full contact with the TMSs. However, significantly higher $R^{*}$ values were obtained with $R_{1} \beta_{1}$ and $R_{1} \beta_{2}$ (greater than $46 \%$, and up to $84 \%$ ), implying that air was trapped at the interface. Indeed, the viscoelastic properties of the gels may determine their deformation capacity and thus their ability to fill the surface asperities of the TMS. The degree of contact between a rigid rough surface and a smooth deformable surface is determined by the capacity of the soft surface to deform and to mold itself to the asperities of the hard surface [49-51].

Many studies have tackled how material properties affect the US apparent reflection coefficient of rough interfaces [49,50,52]. Even though the rigidity of PVC (around 3-3.3 GPa according to the supplier) may be comparable to that of materials used in those past studies (which ranged from rubbers to metals), working with soft gels with low rigidity $(5-140 \mathrm{kPa}$ ) is an original approach. Moreover, one can be noted that the roughness of the interfaces previously studied $\left(R_{a}\right.$ range: $0.2-2 \mu \mathrm{m}$ and $\beta$ range: $2-25 \mu \mathrm{m}$ ) were very different from those used in our experiments. That said, phenomena similar to those in our study were recently examined in work looking at the osseointegration of bone implants using US methods [53]. The researchers investigated the reflection of US waves at an interface between a rough titanium surface and a mixture of bony tissue and soft fibrous tissue. Their numerical simulations revealed that the energy reflected by the rough titanium surface increased as a function of the proportion of soft fibrous tissue in contact with implant asperities. This pattern can be explained by the fact that there is a greater gap in acoustic properties when soft tissues are in contact with the implant surface, as compared to when the implant is in full contact with bone tissue. Along similar lines, we found here that, at the interface between the TMS and the food gels, $R *$ increased as a function of the proportion of air trapped in the asperities of the PVC (and, conversely, decreased as a function of the degree of contact between the food gels and the TMS). The gap in acoustic impedance is indeed greater between the PVC and the air than between the PVC and the food gels. When the PVC was in contact with a greater proportion of food, energy reflection was lower $\left(R^{*}<34 \%\right)$; when the PVC was in contact with a greater proportion of air, energy reflection was much higher (due to the negligible acoustic impedance of air compared to that of PVC). Consequently, the higher the degree of contact between the PVC and the food gels, the lower the value of $R^{*}$. Conversely, the higher the degree of contact between the PVC and the 
Table 4


surface roughness and lubrication. Letters indicate mean values significantly differing among the four levels of lubrication.

\begin{tabular}{|c|c|c|c|c|c|}
\hline & & Lubrication leve & & & \\
\hline Surface roughness & Gel & Dry & Low & Medium & High \\
\hline \multirow[t]{4}{*}{$R_{o} \beta_{o}$} & $A g_{0.3}$ & $33.3 \pm 0.2^{\mathrm{a}}$ & - & - & $33.2 \pm 0.5^{\mathrm{a}}$ \\
\hline & $G e_{3.5}$ & $33.4 \pm 0.7^{\mathrm{a}}$ & - & - & $35.1 \pm 2.3^{\mathrm{a}}$ \\
\hline & $G e_{7}$ & $33.2 \pm 3.7^{\mathrm{a}}$ & $34.7 \pm 3.4^{\mathrm{a}}$ & $38.3 \pm 4.2^{\mathrm{a}}$ & $33.5 \pm 3.7^{\mathrm{a}}$ \\
\hline & $G e_{7} T$ & $23.7 \pm 12.8^{\mathrm{a}}$ & $31.4 \pm 0.8^{\mathrm{a}}$ & $31.0 \pm 0.6^{\mathrm{a}}$ & $23.2 \pm 14.4^{\mathrm{a}}$ \\
\hline \multirow[t]{8}{*}{$R_{1} \beta_{1}$} & $\mathrm{Ag}_{0.3}$ & $45.9 \pm 3.4^{\mathrm{a}}$ & $33.9 \pm 2.2^{\mathrm{b}}$ & $34.5 \pm 1.3^{\mathrm{b}}$ & $35.2 \pm 0.9^{b}$ \\
\hline & $A g_{1.8}$ & $84.8 \pm 3.3^{\mathrm{a}}$ & $76.6 \pm 5.7^{\mathrm{a}}$ & $69.3 \pm 27.0^{\mathrm{a}, \mathrm{b}}$ & $37.1 \pm 1.9^{\mathrm{b}}$ \\
\hline & $G e_{3.5}$ & $66.2 \pm 2.5^{\mathrm{a}}$ & $52.1 \pm 5.8^{\mathrm{b}}$ & $48.6 \pm 2.8^{\mathrm{b}}$ & $35.4 \pm 1.6^{c}$ \\
\hline & $G e_{7}$ & $80.3 \pm 1.0^{\mathrm{a}}$ & $83.4 \pm 9.4^{\mathrm{a}}$ & $63.2 \pm 3.1^{\mathrm{b}}$ & $33.9 \pm 1.8^{\mathrm{c}}$ \\
\hline & $G e_{7} T$ & $81.4 \pm 3.8^{\mathrm{a}}$ & $63.7 \pm 15.3^{\mathrm{b}}$ & $40.6 \pm 8.4^{\mathrm{c}}$ & $35.7 \pm 2.1^{\mathrm{c}}$ \\
\hline & $\mathrm{Ag}_{0.3} \mathrm{Ge}_{3.5}$ & $74.2 \pm 3.9^{\mathrm{a}}$ & $61.4 \pm 9.8^{\mathrm{a}, \mathrm{b}}$ & $54.9 \pm 8.7^{\mathrm{b}}$ & $34.3 \pm 0.9^{c}$ \\
\hline & $A g_{0.7} G_{5.85}$ & $84.3 \pm 0.7^{\mathrm{a}}$ & $65.6 \pm 11.1^{\mathrm{b}}$ & $60.6 \pm 20.4^{b}$ & $35.4 \pm 1.4^{c}$ \\
\hline & $A g_{1} G e_{0.75}$ & $70.1 \pm 3.6^{\mathrm{a}}$ & $68.8 \pm 8.4^{\mathrm{a}}$ & $59.4 \pm 22.8^{\mathrm{a}, \mathrm{b}}$ & $35.2 \pm 1.5^{\mathrm{b}}$ \\
\hline \multirow[t]{4}{*}{$R_{1} \beta_{2}$} & $\mathrm{Ag}_{0.3}$ & $37.8 \pm 3.2^{\mathrm{a}}$ & - & - & $35.0 \pm 0.9^{\mathrm{a}}$ \\
\hline & $G e_{3.5}$ & $53.6 \pm 1.8^{\mathrm{a}}$ & - & - & $37.6 \pm 3.3^{\mathrm{b}}$ \\
\hline & $\mathrm{Ge}_{7}$ & $68.1 \pm 2.3^{\mathrm{a}}$ & $59.3 \pm 8.2^{\mathrm{a}}$ & $42.7 \pm 2.5^{\mathrm{b}}$ & $34.0 \pm 1.1^{\mathrm{c}}$ \\
\hline & $G e_{7} T$ & $63.6 \pm 0.4^{\mathrm{a}}$ & $53.5 \pm 1.0^{\mathrm{b}}$ & $36.3 \pm 2.0^{c}$ & $34.7 \pm 0.5^{c}$ \\
\hline
\end{tabular}

air, the higher the value of $R^{*}$. This mechanism explains why $R^{*}$ was higher for $R_{1} \beta_{1}$ and $R_{1} \beta_{2}$ with their rougher surfaces than for $R_{0} \beta_{0}$ with its smooth surface.

Interestingly, $\beta$ was greater for $R_{1} \beta_{2}$ than for $R_{1} \beta_{1}$, and $R^{*}$ was subsequently lower. Functionally speaking, increasing $\beta$ is equivalent to horizontally stretching surface asperities, leading to peaks and valleys with the same heights/depths but with different slopes ( $\beta$ tends to infinity for smooth surfaces). Thus, as $\beta$ increases, it is less necessary for food to be able to mold itself to surface asperities, leading to a higher degree of contact $[52,54]$ and lower values of $R^{*}$.

\subsection{Impact of food properties on $R^{*}$}

As seen in the previous section, the ability of the food gels to mold themselves to the asperities of TMSs underlies variation in the apparent reflection coefficient. The aim of this section is therefore to discuss the impact of food properties on $R^{*}$.

Depending on their agar or gelatin concentrations, the food gels in this study displayed either a continuous gelatin phase with dispersed agar (gelatin-dominant gels) or a continuous agar phase with dispersed gelatin (agar-dominant gels) [55]. Fig. 3.a shows the $R^{*}$ values at one roughness level, $R_{1} \beta_{1}$, for the different food gels. The gels were split into two groups (gelatin-dominant and agar-dominant) and were ranked by increasing Young's modulus values (see Fig. 3b). The relationship between $R^{*}$ and Young's modulus for the two gel types was also examined (Fig. 4). When the two gel types were considered separately, an increase in $R^{*}$ was observed with increasing Young's modulus values, suggesting that the degree of contact between the food gels and the asperities of the TMSs was decreasing. Consequently, the response of the food gel to its own weight probably significantly contributed to mitigating the gap in acoustic impedance at the interface.

However, other results (Fig. 4) suggest that the Young's modulus values could not entirely explain variation in $R^{*}$. For instance, even if $\mathrm{Ge}_{3.5}$ and $\mathrm{Ag}_{0.3}$ had similar Young's modulus values (around $3 \mathrm{kPa}$ ), $R^{*}$ was $20 \%$ larger for the gelatin-dominant gel. Similarly, $A g_{0.7} \mathrm{Ge}_{5.85}$ and $A g_{1.8}$ resulted in comparable $R^{*}$ values $(\sim 85 \%)$, but the Young's modulus value of the agar-dominant gel was almost four times higher.

The range of the Young's modulus values that we observed indicates that food adhesive energy could also be a contributing factor [56]. The interaction between the food gels and the TMSs was likely mediated by a combination of adhesive and elastic forces, which could promote and moderate, respectively, the food's penetration of the surface asperities $[51,57,58]$. The relative strengths of the adhesive and elastic forces may have been influenced by the structure of the two gel types. The
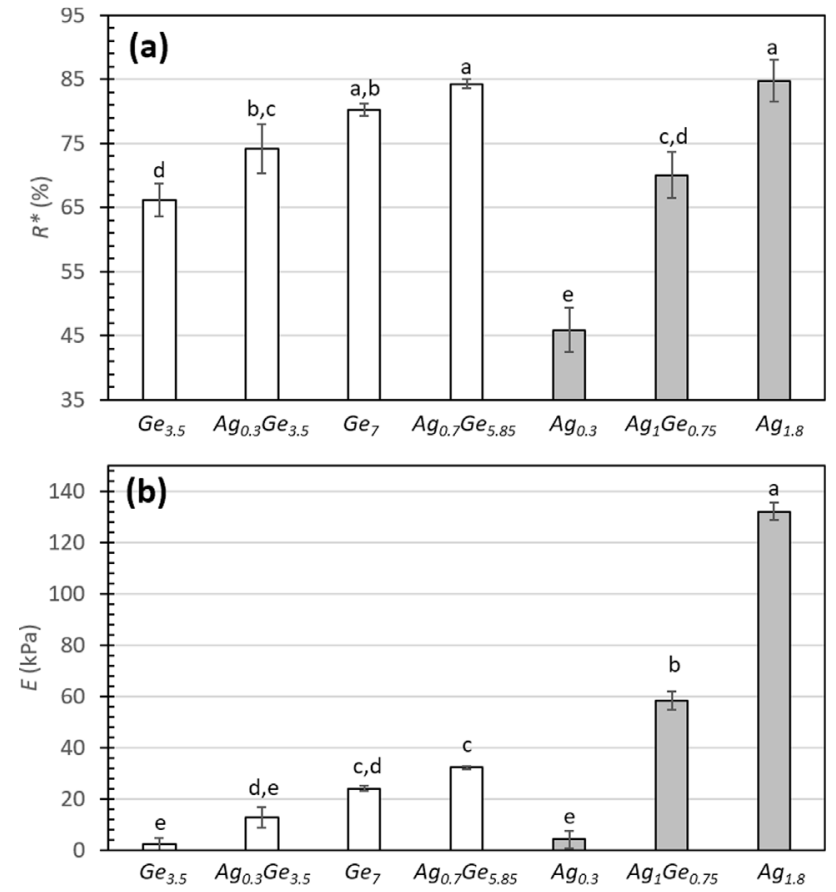

Fig. 3. Mean values and standard deviations of (a) the apparent reflection coefficient at the interface between food gels and a TMS of intermediate roughness $\left(R_{1} \beta_{1}\right)$ under dry lubrication condition and of (b) Young's modulus for the different food gels (white $=$ gelatin-dominant gels, gray = agardominant gels). Letters indicate mean values significantly differing among the four levels of lubrication.

continuous phase of gelatin-dominant products comprises a network of gelatin triple helixes. For agar-dominant products, it is a network of double-helix agar chains. To obtain food products with similar Young's modulus values, lower concentrations of gelling agents are required for agar-dominant versus gelatin-dominant gels. However, as a result, the structure of agar-dominant products is more brittle, with less water bonds to the polymer network. Indeed, agar gels have been shown to display syneresis [59], which is the ability of hydrocolloids to expel water because of interactions between osmotic pressure and elastic forces. In this study, the release of water at the surface of the agardominant gels likely served to improve acoustic coupling properties at the interface between the food gel and the TMS (i.e., acting similarly to 




Fig. 4. Mean values and standard deviations of the apparent reflection coefficient at the interface between food gels and a TMS of intermediate roughness $\left(R_{1} \beta_{1}\right)$ under dry lubrication conditions as a function of Young's modulus for agar- and gelatin-dominant gels (in blue and orange, respectively). (For interpretation of the references to colour in this figure legend, the reader is referred to the web version of this article.)

an acoustic coupling gel), which potentially led to the decrease in $R^{*}$. A recent paper by Santagiuliana et al. (2018) has shown that the amount of serum release under the compression of gels was higher for agar gels than for gelatin gels [15]. They have also shown that for both of them, increasing the concentration in polymer lead to a decrease of the serum release properties. As a result, increasing agar levels could have reduced syneresis and thus increased $R^{*}$ (Fig. 3), in a similar way to Young's modulus.

\subsection{Impact of the lubrication added to the surface on $R^{*}$}

The results discussed thus far are focused solely on the effects of surface roughness and food properties on $R^{*}$ in absence of lubrication. However, it is also important to understand the impact of lubrication in our experimental system, as the results could inform future work on how the salivary film affects the reflection of US waves at food-tongue interface. A few studies (combing analytical and experimental approaches) have successfully used the US apparent reflection coefficient to characterize the thickness of a lubricating oil film trapped between flat sheets of glass [60] or two eccentric aluminum cylinders [61]. However, to our knowledge, no research has looked at a lubricating film trapped between a rough rigid surface and a soft material.

Using real saliva as a lubricant was not feasible because saliva has highly complex and unstable properties (i.e., heterogeneous, nonNewtonian, and viscoelastic [62]). For the sake of simplicity, water was preferred over any of the many saliva substitutes available, such as porcine or bovine mucin solutions [63] and lecithin-based emulsions [64]. There is still debate about the ability of these substitutes to display the rheological behavior of real saliva. Since real saliva is almost entirely composed of water (more than 99\%) [62,65], we can reasonably assume that both the density and the US velocity (and, consequently, the acoustic impedance) in water are very similar to what would be seen in saliva. Furthermore, the acoustic impedance of the different food gels was like that of water (e.g., 1.48 MRay for water and 1.57 MRay for $\mathrm{Ge}_{7}$ [45]). As a consequence, it is unlikely that using water as a lubricant at the contact zone between the TMSs and the food gels resulted in additional interfaces that would generate new echoes in $r f$ signals; instead, it probably behaved as a coupling agent that promoted contact.

Table 4 summarizes the mean values of $R *$ obtained with the different gels at different levels of surface roughness and lubrication.
Statistical results from multiple comparison tests indicate, in each raw, the significant differences between the four levels of lubrication. In absence of lubrication, the results depicted section 3.2 have shown that for $R_{1} \beta_{1}$, various values of $R^{*}$ were reported throughout the different food gels. Different properties of food have been identified as potential drivers of this variability, such as rigidity, adhesive energy, as well as syneresis ability. In Table 4, the results obtained with $R_{1} \beta_{2}$ confirm this tendency: in absence of lubricating film on the TMS, higher values of $R^{*}$ were reported for food gels with higher rigidity (when comparing $\mathrm{Ge}_{7}$ and $\mathrm{Ge}_{7} \mathrm{~T}$ to $\mathrm{Ag}_{0.3}$ and $\mathrm{Ge}_{3.5}$ ) or with lower water release ability (when comparing gelatin dominant sample $\mathrm{Ge}_{3.5}$ with agar dominant sample $A g_{0.3}$ ). The results in Table 4 also show that for the high lubrication level, $R^{*}$ values were low and contained in narrow intervals: for each food gel-roughness pair, the high lubrication level (i.e., water film around $1 \mathrm{~mm}$ thick) systematically yielded the lowest value of $R^{*}$ (generally between 33 and 37\%). For their part, the low and medium lubrication conditions were systematically found to lead to intermediate behaviors. Even though the standard deviations were higher for low and medium conditions compared to dry and high ones (likely due to spreading heterogeneity of the lubrication film), a systematic decrease of $R *$ was observed when moving successively from the lowest to highest lubrication level. When comparing the different food products, one can note that the higher the value of $R^{*}$ in dry condition, the higher the amplitude of the decrease of $R^{*}$ through lubrication. Thicker water films optimized the coupling between the food gels and the rougher TMSs. It should be noted that for $R_{0} \beta_{0}$, lubrication did not lead to a statistically significant decrease in $R^{*}$ because there was already maximal coupling under dry conditions (see Table 4).

In past research, salivary film thickness has been measured in vivo on the anterior part of the tongues of healthy volunteers under normal conditions $(54 \mu \mathrm{m})$ and after drying the tongue with cotton gauze $(12.3 \mu \mathrm{m})$ [66]. Consequently, the low $(15 \mu \mathrm{m})$ and medium $(40 \mu \mathrm{m})$ lubrication levels in this study were more consistent with physiological reality than was the high lubrication level. For all the food gels, the low and medium lubrication levels helped describe the decline in $R^{*}$ values from the dry to high levels (see Table 4). Similar results have been observed in research examining variation in the apparent reflection coefficient when a droplet of water was deposited on a rough hydrophobic surface (made of silicon micropillars) $[67,68]$. The amplitude of the reflected acoustic wave was measured and calibrated based on reference values obtained in air (as it is here). The apparent reflection coefficient was seen to decrease as water droplets moved from the Cassie state (on the top of the asperities) to the Wenzel state (inside the asperities). The results of this study confirm that our US method can characterize this transition.

The food gels $G e_{7}$ and $G e_{7 t}$ and the surface $R_{1} \beta_{1}$ were chosen to illustrate the effect of surface lubrication on $R^{*}$ values (Fig. 5). These two food gels had identical gelatin concentrations (7\%) and differed only in the presence/absence of an emulsifying agent (Tween 20). The objective was to explore the impact of food wettability on the interaction with the lubricating film. Measurements of the contact angle with the water revealed that wettability was extremely high for $\mathrm{Ge}_{7 \mathrm{t}}$, with its contact angle of $0^{\circ}$; in contrast, the contact angle was $40 \pm 12^{\circ}$ for $\mathrm{Ge}_{7}$. Under dry conditions, the two food gels had similar $R^{*}$ values $\left(G e_{7 t}: 80.3 \%\right.$ and $G e_{7}: 81.4 \%$ ), even though the Young's modulus values were slightly higher for $G e_{7 t}$ than for $G e_{7}$. This similarity suggests that contact conditions at the interface between TMS and food gel were similar as well. At high lubrication levels, the two food gels also had similar values of $R^{*}\left(G_{7}: 33.9 \%\right.$ and $\left.G e_{7}: 35.7 \%\right)$, which seems to reflect that there was complete, homogeneous contact. However, the results for the two intermediate lubrication levels show that the two food gels displayed different patterns for the decline in $R^{*}$. Indeed, $R^{*}$ decreased more dramatically for $\mathrm{Ge}_{7 t}$ than for $\mathrm{Ge}_{7}$, at both the low lubrication level (63.7 and $83.4 \%$, respectively) and the medium lubrication level (40.6 and $63.2 \%$, respectively). This finding suggests that, when the lubricant is not present in excess, greater food 


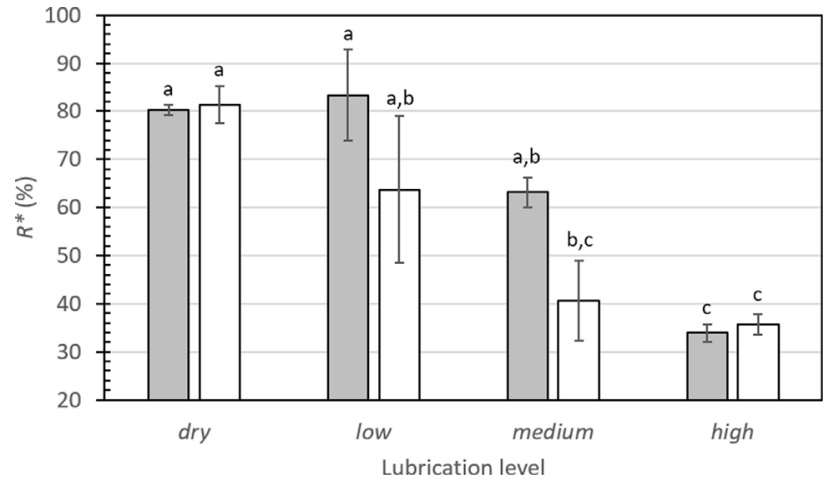

Fig. 5. Mean values and standard deviations of the apparent reflection coefficient at the interface between food gels and a TMS of intermediate roughness $\left(R_{1} \beta_{1}\right)$ across the four lubrication levels for the gels $\mathrm{Ge}_{7}$ (gray) and $\mathrm{Ge}_{7} T$ (white). Letters indicate mean values significantly differing among the four lubricating conditions (Newman-Keuls, $\mathrm{p}<0.05$ ).

wettability can promote film homogeneity across the interface.

In summary, while lubricant level plays a key role, the wettability of the media located on either side of the interface also likely influences the values of the apparent reflection coefficient.

\section{Conclusions}

This study investigated whether a US technique could be used to examine the deposit of food gels on TMSs. These non-deformable surfaces were used to study the impact of surface roughness and lubrication on $R^{*}$ for different food gels.

Our findings show that the apparent reflection coefficient is influenced by differences in surface roughness (both $R_{a}$ and $\beta$ ); food gel type (rigidity, tendency towards syneresis, and wettability); and lubrication level. All these parameters mechanistically interacted with the ability of food gels to mold themselves to the surface asperities of the TMSs, which resulted in a decrease in the apparent reflection coefficient (i.e., due to the decrease in the acoustic impedance gap at the interface). In the future, the US method that we used could greatly facilitate the indirect estimation of the contact area between foods and the tongue surface. Such estimates would be highly useful in clarifying the mechanisms underlying texture perception $[3,69]$, which intervene early on in oral processing. For example, the perception of firmness is due to the transmission of mechanical stresses to the mechanoreceptors in the vicinity of tongue asperities, and the perception of moistness is due to the interactions of food and the salivary film with tongue asperities. Finally, this US method could also serve to characterize tongue surface ratios, which could improve predictions about how sapid compounds diffuse towards the tongue's taste receptors.

To increase the applicability of this work, many physiological issues will need to be addressed in subsequent studies. Future work should focus on enhancing the real-time potential of the US method and on progressively accounting for tongue motility-starting with a compression test before gradually striving for realistic tongue movement. Other priorities should include accounting for the deformability of tongue tissue and the rheological complexity of real saliva. Moreover, a broader diversity of more realistic food types should be considered (e.g., with irregular shapes, heterogeneities, and air incorporated), and the changing properties of the food bolus during oral processing should be examined (e.g., particle size reduction, saliva incorporation).

\section{Declaration of Competing Interest}

The authors declare that they have no known competing financial interests or personal relationships that could have appeared to influence the work reported in this paper.

\section{Acknowledgments}

We wish to acknowledge Jean-Luc Gennisson for the detailed advice he provided on the ultrasound analyses. We are grateful to David Forest, Claire Juguet, Raphaëlle Ponthieux, and Gabriel Debar for their crucial technical support. We also warmly thank Rohit Srivastava and Jessica Pearce-Duvet for their precious help in proofreading the manuscript.

This work was financially supported by (i) the IDI 2015 project funded by IDEX Paris Saclay (ANR-11-IDEX-0003-02), (ii) the PLUS project funded by the French National Institute for Agricultural Research (INRA, 2015), and (iii) the QUSToFood project funded by the French National Research Agency (ANR-17-CE21-004).

\section{References}

[1] J. Chen, Food oral processing-A review, Food Hydrocoll. 23 (2009) 1-25, https:// doi.org/10.1016/j.foodhyd.2007.11.013.

[2] H. Koç, C.J. Vinyard, G.K. Essick, E.A. Foegeding, Food oral processing: conversion of food structure to textural perception, Annu. Rev. Food Sci. Technol. 4 (2013) 237-266, https://doi.org/10.1146/annurev-food-030212-182637.

[3] A.S. Szczesniak, Texture is a sensory property, Food Qual. Prefer. 13 (2002) $215-225$.

[4] T. van Vliet, On the relation between texture perception and fundamental mechanical parameters for liquids and time dependent solids, Food Qual. Prefer. 13 (2002) 227-236.

[5] K.M. Hiiemae, Mechanisms of food reduction, transport and deglutition: how the texture of food affects feeding behavior, J. Texture Stud. 35 (2004) 171-200.

[6] S. Abd-El-Malek, Observations on the morphology of the human tongue, J. Anat. 73 (1939) 201.

[7] Y. Kajee, J.-P.V. Pelteret, B.D. Reddy, The biomechanics of the human tongue: the biomechanics of the human tongue, Int. J. Numer. Methods Biomed. Eng. 29 (2013) 492-514, https://doi.org/10.1002/cnm.2531.

[8] J.L. Miller, K.L. Watkin, M.F. Chen, Muscle, adipose, and connective tissue variations in intrinsic musculature of the adult human tongue, J. Speech Lang. Hear. Res. 45 (2002) 51-65, https://doi.org/10.1044/1092-4388(2002/004).

[9] T.H. Shawker, B.C. Sonies, M. Stone, Soft tissue anatomy of the tongue and floor of the mouth: an ultrasound demonstration, Brain Lang. 21 (1984) 335-350, https:// doi.org/10.1016/0093-934X(84)90056-7.

[10] E. Lauga, C.J. Pipe, B. Le Révérend, Sensing in the mouth: a model for filiform papillae as strain amplifiers, Front. Phys. 4 (2016), https://doi.org/10.3389/fphy. 2016.00035.

[11] H. Ranc, C. Servais, P.-F. Chauvy, S. Debaud, S. Mischler, Effect of surface structure on frictional behaviour of a tongue/palate tribological system, Tribol. Int. 39 (2006) 1518-1526, https://doi.org/10.1016/j.triboint.2006.01.017.

[12] J.R. Stokes, M.W. Boehm, S.K. Baier, Oral processing, texture and mouthfeel: From rheology to tribology and beyond, Curr. Opin. Colloid Interface Sci. 18 (2013) 349-359, https://doi.org/10.1016/j.cocis.2013.04.010.

[13] M. Bukowska, G.K. Essick, M. Trulsson, Functional properties of low-threshold mechanoreceptive afferent in the human labial mucosa, Exp. Brain Res. 201 (2010) 59-64, https://doi.org/10.1007/s00221-009-2005-0.

[14] N. Rommel, S. Hamdy, Oropharyngeal dysphagia: manifestations and diagnosis, Nat. Rev. Gastroenterol. Hepatol. 13 (2016) 49-59, https://doi.org/10.1038/ nrgastro.2015.199.

[15] M. Santagiuliana, B. Piqueras-Fiszman, E. van der Linden, M. Stieger, E. Scholten, Mechanical properties affect detectability of perceived texture contrast in heterogeneous food gels, Food Hydrocoll. 80 (2018) 254-263, https://doi.org/10.1016/j. foodhyd.2018.02.022.

[16] M. Santagiuliana, M. Christaki, B. Piqueras-Fiszman, E. Scholten, M. Stieger, Effect of mechanical contrast on sensory perception of heterogeneous liquid and semisolid foods, Food Hydrocoll. 83 (2018) 202-212, https://doi.org/10.1016/j. foodhyd.2018.04.046.

[17] E.A. Foegeding, M.A. Drake, Invited review: sensory and mechanical properties of cheese texture, J. Dairy Sci. 90 (2007) 1611-1624, https://doi.org/10.3168/jds. 2006-703.

[18] M. Panouillé, A. Saint-Eve, I. Souchon, Instrumental methods for bolus characterization during oral processing to understand food perceptions, Curr. Opin. Food Sci. 9 (2016) 42-49, https://doi.org/10.1016/j.cofs.2016.05.002.

[19] A. Saint-Eve, M. Panouillé, C. Capitaine, I. Déléris, I. Souchon, Dynamic aspects of texture perception during cheese consumption and relationship with bolus properties, Food Hydrocoll. 46 (2015) 144-152, https://doi.org/10.1016/j.foodhyd. 2014.12.015.

[20] S. Camacho, V. van Riel, C. de Graaf, F. van de Velde, M. Stieger, Physical and sensory characterizations of oral coatings of oil/water emulsions, J. Agric. Food Chem. 62 (2014) 5789-5795, https://doi.org/10.1021/jf5010868.

[21] J.S. Chen, Food oral processing - a review, Food Hydrocoll. 23 (2009) 1-25, https://doi.org/10.1016/j.foodhyd.2007.11.013.

[22] T.S. Awad, H.A. Moharram, O.E. Shaltout, D. Asker, M.M. Youssef, Applications of ultrasound in analysis, processing and quality control of food: a review, Food Res. Int. 48 (2012) 410-427, https://doi.org/10.1016/j.foodres.2012.05.004.

[23] D.J. McClements, S. Gunasekaran, Ultrasonic characterization of foods and drinks: 
principles, methods, and applications, Crit. Rev. Food Sci. Nutr. 37 (1997) 1-46, https://doi.org/10.1080/10408399709527766.

[24] C. Javanaud, Applications of ultrasound to food systems, Ultrasonics 26 (1988) 117-123, https://doi.org/10.1016/0041-624X(88)90001-7.

[25] B. Richard, M. Toubal, J.-F. Le Page, G. Nassar, E. Radziszewski, B. Nongaillard, P. Debreyne, P. Schuck, R. Jeantet, G. Delaplace, Ultrasound tests in a stirred vessel to evaluate the reconstitution ability of dairy powders, Innov. Food Sci. Emerg. Technol. 16 (2012) 233-242, https://doi.org/10.1016/j.ifset.2012.06.007.

[26] P. Pallav, D.A. Hutchins, T.H. Gan, Air-coupled ultrasonic evaluation of food materials, Ultrasonics. 49 (2009) 244-253, https://doi.org/10.1016/j.ultras.2008.09. 002.

[27] D. Mcclements, M. Povey, M. Jury, E. Betsanis, Ultrasonic characterization of a food emulsion, Ultrasonics 28 (1990) 266-272, https://doi.org/10.1016/0041-624X(90) 90093-4.

[28] H. Ogasawara, K. Mizutani, T. Ohbuchi, T. Nakamura, Acoustical experiment of yogurt fermentation process, Ultrasonics. 44 (2006) E727-E730, https://doi.org/ 10.1016/j.ultras.2006.05.084.

[29] C. Letang, M. Piau, C. Verdier, L. Lefebvre, Characterization of wheat-flour-water doughs: a new method using ultrasound, Ultrasonics 39 (2001) 133-141, https:// doi.org/10.1016/S0041-624X(00)00058-5.

[30] C.A. Kelsey, F.D. Minifie, Ultrasonic scans of the tongue, J. Acoust. Soc. Am. 49 (1971) 133-134, https://doi.org/10.1121/1.1975854.

[31] M. Stone, A guide to analysing tongue motion from ultrasound images, Clin. Linguist. Phon. 19 (2005) 455-501, https://doi.org/10.1080/ 02699200500113558.

[32] C.-Y. Chien, J.-C. Chen, C.-H. Chang, C.-C. Huang, Tracking dynamic tongue motion in ultrasound images for obstructive sleep apnea, Ultrasound Med. Biol. (2017), https://doi.org/10.1016/j.ultrasmedbio.2017.08.001.

[33] C.-K. Weng, J.-W. Chien, P.-Y. Lee, C.-C. Huang, Implementation of a wearable ultrasound device for the overnight monitoring of tongue base deformation during obstructive sleep apnea events, Ultrasound Med. Biol. 43 (2017) 1639-1650, https://doi.org/10.1016/j.ultrasmedbio.2017.04.004.

[34] M.-Y. Hsiao, Y.-C. Chang, W.-S. Chen, H.-Y. Chang, T.-G. Wang, Application of ultrasonography in assesing oropharyngeal dysphagia in stroke patients, Ultrasound Med. Biol. 38 (2012) 1522-1528, https://doi.org/10.1016/j.ultrasmedbio.2012.04. 017.

[35] J.-H. Kim, M.-S. Kim, Lateral pharyngeal wall motion analysis using ultrasonography in stroke patients with dysphagia, Ultrasound Med. Biol. 38 (2012) 2058-2064, https://doi.org/10.1016/j.ultrasmedbio.2012.07.028.

[36] G. Chi-Fishman, Quantitative lingual, pharyngeal and laryngeal ultrasonography in swallowing research: a technical review, Clin. Linguist. Phon. 19 (2005) 589-604, https://doi.org/10.1080/02699200500113996.

[37] R.A. de Wijk, F. Wulfert, J.F. Prinz, Oral processing assessed by M-mode ultrasound imaging varies with food attribute, Physiol. Behav. 89 (2006) 15-21, https://doi. org/10.1016/j.physbeh.2006.05.021.

[38] S. Galén, P.-G. Jost-Brinkmann, B-mode and M-mode ultrasonography of tongue movements during swallowing, J. Orofac. Orthop. Fortschritte Kieferorthopädie. 71 (2010) 125-135, https://doi.org/10.1007/s00056-010-9928-8.

[39] Z. Gao, M. Taniwaki, H. Shimada, S. Ishihara, M. Nakauma, T. Funami, K. Kohyama, Ultrasound analysis of the effects of food bolus volume on tongue movement at the initiation of swallowing, J. Texture Stud. 44 (2013) 387-396, https://doi.org/10. 1111/jtxs.12026.

[40] Z. Gao, K. Kohyama, Ultrasound pulsed wave Doppler imaging of the esophagus illustrates the effects of water volume on bolus kinematics, J. Texture Stud. 45 (2014) 335-343, https://doi.org/10.1111/jtxs.12077.

[41] C.-L. Peng, P.-G. Jost-Brinkmann, R.-R. Miethke, C.-T. Lin, Ultrasonographic measurement of tongue movement during swallowing, J. Ultrasound Med. 19 (2000) 15-20.

[42] Z. Gao, S. Nakao, S. Ishihara, T. Funami, K. Kohyama, A pilot study on ultrasound elastography for evaluation of mechanical characteristics and oral strategy of gels, J. Texture Stud. 47 (2016) 152-160, https://doi.org/10.1111/jtxs.12170.

[43] Z. Gao, S. Ishihara, S. Nakao, F. Hayakawa, T. Funami, K. Kohyama, Texture evaluation of soft gels with different fracture strains using an artificial tongue, J. Texture Stud. 47 (2016) 496-503, https://doi.org/10.1111/jtxs.12210.

[44] M. Devezeaux de Lavergne, M. van Delft, F. van de Velde, M.A.J.S. van Boekel, M. Stieger, Dynamic texture perception and oral processing of semi-solid food gels: Part 1: Comparison between QDA, progressive profiling and TDS, Food Hydrocoll. 43 (2015) 207-217, https://doi.org/10.1016/j.foodhyd.2014.05.020.

[45] M. Mantelet, M. Panouille, F. Boue, V. Bosc, F. Restagno, I. Souchon, V. Mathieu, Impact of sol-gel transition on the ultrasonic properties of complex model foods: Application to agar/gelatin gels and emulsion filled gels, Food Hydrocoll. 87 (2019) 506-518, https://doi.org/10.1016/j.foodhyd.2018.08.021.

[46] E.S. Gadelmawla, M.M. Koura, T.M.A. Maksoud, I.M. Elewa, H.H. Soliman, Roughness parameters, J. Mater. Process. Technol. 123 (2002) 133-145, https:// doi.org/10.1016/S0924-0136(02)00060-2.
[47] B. Drinkwater, P. Cawley, Measurement of the frequency dependence of the ultrasonic reflection coefficient from thin interface layers and partially contacting interfaces, Ultrasonics 35 (1997) 479-488, https://doi.org/10.1016/S0041-624X(97) 00072-3.

[48] N. Uemori, Y. Kakinoki, J. Karaki, H. Kakigawa, New method for determining surface roughness of tongue, Gerodontology 29 (2012) 90-95, https://doi.org/10. 1111/j.1741-2358.2011.00509.x.

[49] M. Gonzalez-Valadez, A. Baltazar, R.S. Dwyer-Joyce, Study of interfacial stiffness ratio of a rough surface in contact using a spring model, Wear 268 (2010) 373-379, https://doi.org/10.1016/j.wear.2009.08.022.

[50] K. Hodgson, R.S. Dwyer-Joyce, B.W. Drinkwater, Ultrasound as an experimental tool for investigating engineering contacts, Tribologia 19 (2000) 9-17.

[51] B.N.J. Persson, O. Albohr, U. Tartaglino, A.I. Volokitin, E. Tosatti, On the nature of surface roughness with application to contact mechanics, sealing, rubber friction and adhesion, J. Phys. Condens. Matter. 17 (2005) R1-R62, https://doi.org/10. 1088/0953-8984/17/1/R01.

[52] B. Drinkwater, R. Dwyer-Joyce, P. Cawley, A study of the transmission of ultrasound across solid-rubber interfaces, J. Acoust. Soc. Am. 101 (1997) 970-981.

[53] Y. Hériveaux, V.-H. Nguyen, G. Haïat, Reflection of an ultrasonic wave on the boneimplant interface: a numerical study of the effect of the multiscale roughness, J. Acoust. Soc. Am. 144 (2018) 488-499, https://doi.org/10.1121/1.5046524.

[54] K. Kendall, D. Tabor, An ultrasonic study of the area of contact between stationary and sliding surfaces, Proc. R. Soc. Math. Phys. Eng. Sci. 323 (1971) 321-340, https://doi.org/10.1098/rspa.1971.0108.

[55] H. McEvoy, S.B. Ross-Murphy, A.H. Clark, Large deformation and ultimate properties of biopolymer gels: 2. Mixed gel systems, Polymer 26 (1985) 1493-1500, https://doi.org/10.1016/0032-3861(85)90082-5.

[56] K.R. Shull, Contact mechanics and the adhesion of soft solids, Mater. Sci. Eng. R Rep. 36 (2002) 1-45, https://doi.org/10.1016/S0927-796X(01)00039-0.

[57] L. Dies, F. Restagno, R. Weil, L. Léger, C. Poulard, Role of adhesion between asperities in the formation of elastic solid/solid contacts, Eur. Phys. J. E 38 (2015), https://doi.org/10.1140/epje/i2015-15130-4.

[58] C. Poulard, F. Restagno, R. Weil, L. Léger, Mechanical tuning of adhesion through micro-patterning of elastic surfaces, Soft Matter. 7 (2010), https://doi.org/10. 1039/c0sm01099e.

[59] S. Boral, A. Saxena, H.B. Bohidar, Syneresis in agar hydrogels, Int. J. Biol. Macromol. 46 (2010) 232-236, https://doi.org/10.1016/j.ijbiomac.2009.12.008.

[60] T. Reddyhoff, S. Kasolang, R.S. Dwyer-Joyce, B.W. Drinkwater, The phase shift of an ultrasonic pulse at an oil layer and determination of film thickness, Proc. Inst. Mech. Eng. Part J J. Eng. Tribol. 219 (2005) 387-400, https://doi.org/10.1243/ $135065005 X 34044$.

[61] R.S. Dwyer-Joyce, B.W. Drinkwater, C.J. Donohoe, The measurement of lubricant-film thickness using ultrasound, Proc. R. Soc. Lond. Math. Phys. Eng. Sci. 459 (2003) 957-976, https://doi.org/10.1098/rspa.2002.1018.

[62] S.P. Humphrey, R.T. Williamson, A review of saliva: Normal composition, flow, and function, J. Prosthet. Dent. 85 (2001) 162-169, https://doi.org/10.1067/mpr. 2001.113778.

[63] A. Sarkar, K.K.T. Goh, H. Singh, Colloidal stability and interactions of milk-proteinstabilized emulsions in an artificial saliva, Food Hydrocoll. 23 (2009) 1270-1278, https://doi.org/10.1016/j.foodhyd.2008.09.008.

[64] S.M. Hanning, T. Yu, D.S. Jones, G.P. Andrews, J.A. Kieser, N.J. Medlicott, Lecithinbased emulsions for potential use as saliva substitutes in patients with xerostomia viscoelastic properties, Int. J. Pharm. 456 (2013) 560-568, https://doi.org/10. 1016/j.ijpharm.2013.08.009.

[65] J.H.H. Bongaerts, D. Rossetti, J.R. Stokes, The lubricating properties of human whole saliva, Tribol. Lett. 27 (2007) 277-287, https://doi.org/10.1007/s11249007-9232-y.

[66] R. Pramanik, S.M. Osailan, S.J. Challacombe, D. Urquhart, G.B. Proctor, Protein and mucin retention on oral mucosal surfaces in dry mouth patients, Eur. J. Oral Sci. 118 (2018) 245-253, https://doi.org/10.1111/j.1600-0722.2010.00728.x.

[67] R. Dufour, N. Saad, J. Carlier, P. Campistron, G. Nassar, M. Toubal, R. Boukherroub, V. Senez, B. Nongaillard, V. Thomy, Acoustic tracking of cassie to wenzel wetting transitions, Langmuir 29 (2013) 13129-13134, https://doi.org/10.1021/ la402481b.

[68] N. Saad, B. Merheb, G. Nassar, P. Campistron, J. Carlier, M. Ajaka, B. Nongaillard, Characterization of the state of a droplet at a micro-textured silicon wafer using a finite difference time-domain (FDTD) modeling method, IOP Conf. Ser. Mater. Sci. Eng. 42 (2012) 012052, https://doi.org/10.1088/1757-899X/42/1/012052.

[69] G. Ares, D. GonçAlvez, C. PéRez, G. ReolóN, N. Segura, P. Lema, A. GáMbaro, Influence of gelatin and starch on the instrumental and sensory texture of stirred yogurt, Int. J. Dairy Technol. 60 (2007) 263-269, https://doi.org/10.1111/j.1471 0307.2007.00346.x.

[70] R. Pramanik, S.M. Osailan, S.J. Challacombe, D. Urquhart, G.B. Proctor, Protein and mucin retention on oral mucosal surfaces in dry mouth patients, Eur. J. Oral Sci. 118 (2010) 245-253. 